\title{
A note on super Koszul complex and the Berezinian
}

\author{
Simone Noja ${ }^{1}$ (D) $\cdot$ Riccardo $\operatorname{Re}^{3,4}$
}

Received: 28 January 2021 / Accepted: 3 May 2021 / Published online: 27 May 2021

(c) The Author(s) 2021

\begin{abstract}
We construct the super Koszul complex of a free supercommutative $A$-module $V$ of rank $p \mid q$ and prove that its homology is concentrated in a single degree and it yields an exact resolution of $A$. We then study the dual of the super Koszul complex and show that its homology is concentrated in a single degree as well and isomorphic to $\Pi^{p+q} A$, with $\Pi$ the parity changing functor. Finally, we show that, given an automorphism of $V$, the induced transformation on the only non-trivial homology class of the dual of the super Koszul complex is given by the multiplication by the Berezinian of the automorphism, thus relating this homology group with the Berezinian module of $V$.
\end{abstract}

Keywords Superalgebra $\cdot$ Koszul Complex $\cdot$ Berezinian $\cdot$ Supergeometry

Mathematics Subject Classification $17 \mathrm{~A} 70 \cdot 16 \mathrm{E} 30 \cdot 16 \mathrm{E} 40 \cdot 58 \mathrm{~A} 50$

\section{Introduction}

The definition of the Koszul Complex, whose first introduction as an example of a complex of free modules over a commutative ring $A$ dates back to Hilbert, marks the advent of homological methods in commutative algebra in the early 1950s. Since it is a resolution of $k=A / m$ with $(A, m)$ a regular local ring, or of $R=S /\left(x_{0}, \ldots, x_{r}\right)$, with $S=R\left[x_{0}, \ldots, x_{r}\right]$, it allowed the computation of derived functors like $\operatorname{Tor}^{i}(A, M)$ and $\operatorname{Ext}^{i}(A, M)$ for any $S$-module $M$, hence of important homological invariants-like the projective dimension of a module (or its Koszul homology) — and their relations to more classical concepts, like for example the depth of a module.

Simone Noja

noja@mathi.uni-heidelberg.de

Riccardo Re

riccardo.re@uninsubria.it

1 Universität Heidelberg, Heidelberg, Germany

2 Present Address: Im Neuenheimer Feld 205, 69120 Heidelberg, Germany

3 Università degli Studi dell'Insubria, Como, Italy

4 Present Address: Università degli Studi dell’Insubria, Via Valleggio 11, 22100 Como, Italy 
The Koszul complex may also be constructed in a non-commutative setting, i.e. for any left $A$-module $M$ with $A$ any ring and elements $x_{1}, \ldots, x_{r}$ in $A$, there exists a Koszul complex $K\left(x_{1}, \ldots, x_{r}, M\right)$, when $x_{i}$ are pairwise commuting when viewed as multiplication maps $x_{i}: M \rightarrow M$. In this paper, we consider the case of a commutative superalgebra $S$ and therefore we take the $x_{i}$ 's to be supercommutative. We first provide the construction of the Koszul complex $K\left(x_{1}, \ldots, x_{r}\right)$ in the supercommutative case and then we compute its homology in the universal case $S=A\left[x_{1}, \ldots, x_{r}\right]$, with $A$ supercommutative. In doing so, we revisit the proof that it is a resolution of $A$ as a $S$-module, and then we study the dual complex $K^{*}\left(x_{1}, \ldots, x_{r}\right)$ and calculate its homology, hence computing $\operatorname{Ext}^{i}(A, S)$. In particular, this produces the Berezinian of the free $A$-module $F=\bigoplus_{i} A x_{i}$.

Although it is fair to say that results regarding the Koszul complex in a "super setting" have previously appeared - see [8] -, we are not aware of a complete and detailed treatment of this fundamental construction in the existing superalgebra or supergeometry literature. We take the chance to fill such a gap with the present paper, which provides also a completely selfcontained exposition of the subject. Further, we remark that the supercommutative setting for the Koszul complex includes of course the commutative case, in a way that clearly shows the intimate supercommutative nature of the classical Koszul complex. Moreover, the magical self-duality of the classical Koszul complex-allowing for example for the rich theory of complete intersections or, more generally, Gorenstein rings in commutative algebra -, is put in the right setting within the treatment of the super Koszul complex of this paper, where it is made clear that the dual of a super Koszul complex is not in general isomorphic to the same Koszul complex, but to another one, depending on the numbers of even and odd variables involved. Finally, we stress that the given construction via homology of the super Koszul complex, provides a completely invariant construction of the Berezinian of a free-module, a crucial building block for modern supersymmetric theories in theoretical physics.

The paper is structured as follows. In section two, we establish our conventions and we provide the reader with some definitions and preliminary result. In particular, in Theorem 2.2, we use our superalgebraic setting to compute the homology of the classic Koszul complex, which will be used later on in the paper. In section three, we construct the super Koszul complex of a free supercommutative $A$-module $V$ and we compute its homology in Theorem 3.5. The result is built upon Lemma 3.3 and Lemma 3.4, which compute the homotopy operator of the differential of the super Koszul complex. In this respect, in Remark 3.6 at the end of section three, we address the differences with the classical Koszul homology and we briefly discuss the interesting case of characteristic $p$ in the superalgebraic setting by means on an example. In section four we introduce the dual of the super Koszul complex and we briefly discuss the functoriality of the construction. Then we proceed to compute the homology of the dual of the super Koszul complex in Theorem 4.3, whose proof is based on the ancillary result Theorem 2.2. Finally, in the last section we make contact with the Berezinian module of $V$ : in particular, we prove that given an automorphism of $V$, a representative of the homology of the dual of the super Koszul complex transforms with the inverse of the Berezinian of the automorphism. This allows to identify the (dual of) Berezinian module of $V$ with the only nontrivial homology module of the dual of the super Koszul complex of $V$.

Addendum: soon after this paper appeared as a preprint, Prof. Ogievetsky makes us aware that the super Koszul complex and its dual first appeared in [9], by him and I.B. Penkov—see Corollary 4 therein -, but a detailed treatment has never indeed appeared in the literature. We would like to thank him for pointing out this reference to us. 


\section{Preliminary definitions and the Koszul Complex via Superalgebra}

In this section, we recall some elements of superalgebra that will be used in the following. For a thorough exposition of superalgebra we refer to the classical [8] or the recent [5].

Let $A$ be any superalgebra of characteristic 0 and let $V=A^{p \mid q}$ be a free $A$-supermodule with basis given by $\left\{x_{1}, \ldots, x_{p} \mid \theta_{1}, \ldots, \theta_{q}\right\}$, where the $\mathbb{Z}_{2}$-grading, or parity, reads $\left|x_{i}\right|=0$ and $\left|\theta_{j}\right|=1$ for any $i=1, \ldots, p$ and $j=1, \ldots, q$. We define

$$
R:=\bigoplus_{k \geq 0} R_{k} \quad \text { with } \quad R_{k}:=\operatorname{Sym}^{k} V,
$$

where $\operatorname{Sym}^{k}(\cdot): \mathbf{S M o d}_{A} \rightarrow \mathbf{S M o d}_{A}$ is the (super)symmetric $k$-power functor from the category of $A$-supermodules to itself. Henceforth we will refer to $\operatorname{Sym}^{k}(\cdot)$ simply as the $k$-symmetric product. We observe that $R$ has a structure of a $\mathbb{Z}$-graded $A$-algebra, where the products $R_{i} \otimes R_{j} \rightarrow R_{i+j}$ are induced by the symmetric product, and also of a $\mathbb{Z}_{2}$-graded commutative (or supercommutative) $A$-algebra-we say that it is an $A$-superalgebrawhere the grading is induced by that of $V$.

It is worth noticing that $A$ is a $R$-module thanks to the short exact sequence

$$
0 \longrightarrow I_{1} \longrightarrow R \longrightarrow A \longrightarrow 0
$$

where $I_{1}:=\bigoplus_{k \geq 1} S y m^{k} V$ is the (maximal) ideal of $R$ generated by $V \cong S y m^{1} V \subset R$ and, hence, $A \cong R / I_{1} R$. The ideal $I_{1}$ has the following presentation:

$$
R \otimes \Pi V \stackrel{p}{\longrightarrow} I_{1} \longrightarrow 0
$$

where $\Pi: \mathbf{S M o d}_{A} \rightarrow \mathbf{S M o d}_{A}$ is the parity changing functor, acting on objects by simply reversing their parity. In the (2.3) the (surjective) morphism $p: R \otimes \Pi V \rightarrow I_{1}$ is defined as follows on even and odd generators

$$
\begin{aligned}
& 1 \otimes \pi x_{i} \stackrel{p}{\longmapsto} x_{i}, \\
& 1 \otimes \pi \theta_{i} \stackrel{p}{\longmapsto} \theta_{j} .
\end{aligned}
$$

Notice that $p$ is an $o d d$ morphism, as it reverses parity.

Given $V$ as above we define its dual as $V^{*}:=\operatorname{Hom}_{A}(V, A)$. This defines again a supercommutative $A$-module with parity splitting given by $V^{*}=\operatorname{Hom}_{A}(V, A)_{0} \oplus \operatorname{Hom}_{A}(V, A)_{1}$, i.e. the even and odd $A$-linear maps. For any $i \leq k$ there exists a pairing given by

$$
\begin{aligned}
\langle\cdot, \cdot\rangle: S y m^{i} V^{*} \otimes S^{k} m^{k} V & \longrightarrow S y m^{k-i} V \\
t_{i}^{*} \otimes s_{k} \longmapsto & \left\langle t_{i}^{*}, s_{k}\right\rangle .
\end{aligned}
$$

If $V$ has basis given by $\left\{x_{1}, \ldots, x_{p} \mid \theta_{1}, \ldots, \theta_{q}\right\}$ as above, this can be obtained by considering the dual space $V^{*}$ as the space having basis given by $\left\{\partial_{x_{1}}, \ldots, \partial_{x_{p}} \mid \partial_{\theta_{1}}, \ldots, \partial_{\theta_{q}}\right\}$, so that one has the identification 


$$
\operatorname{Sym}^{i} V^{*}:=\left\{D: \operatorname{Sym}^{k \geq i} V \rightarrow \operatorname{Sym}^{k-i} V: D \text { is a homogeneous operator of order } i\right\} .
$$

Notice in particular that, for $k=i$ one has the duality pairing $\operatorname{Sym}^{k} V^{*} \otimes S y m^{k} V \rightarrow A$.

Notably, the above superalgebraic setting can be used to reinterpret the construction of the "ordinary" Koszul complex (see for example [7] for an extended treatment of the subject), and compute its homology in a very economic and elegant way.

Let us consider indeed a free-module $A^{N}$ for a certain ring or algebra $A$ with a basis given by $\left\{x_{1}, \ldots, x_{N}\right\}$. Then one can construct the free supermodule $A^{N \mid N}=A^{N} \oplus \Pi A^{N}$ with a basis given by $\left\{x_{1}, \ldots, x_{N} \mid y_{1}, \ldots, y_{N}\right\}$, where $\left|x_{i}\right|=0$, i.e. the $x_{i}$ 's are even, and $\left|y_{i}\right|=1$, i.e. the $y_{i}$ 's are odd, for any $i=1, \ldots, N$ : in this sense, the $y_{i}$ 's can be defined as $y_{i}:=\pi x_{i}$, just by changing the parity of the generator of $A^{N}$. Notice that the $x_{i}$ 's and the $y_{i}^{\prime}$ 's are not $A$-linearly dependent.

Here, the supermodule $A^{N \mid N}$ plays the role of $V$ introduced in the above construction, so that $R$ can be written as

$$
R=B \oplus(B \cdot U) \oplus\left(B \cdot \operatorname{Sym}^{2} U\right) \oplus \ldots \oplus\left(B \cdot \operatorname{Sym}^{N} U\right)=B \otimes_{A} \bigoplus_{k=0}^{N} \operatorname{Sym}^{k} U .
$$

where we have defined

$$
B:=A\left[x_{1}, \ldots, x_{N}\right] \text { and } U:=A\left[y_{1}, \ldots, y_{N}\right] .
$$

Notice that, classically, $B$ and $U$ can be seen respectively as the symmetric and exterior algebra over a set of $N$ generators (over $A$ ). Let us define the following multiplication operator on $R$ :

$$
\begin{aligned}
& d: R \longrightarrow R \\
& b \cdot F \longmapsto d(b \cdot F):=\left(\sum_{i=1}^{N} x_{i} \cdot y_{i}\right)(b \cdot F),
\end{aligned}
$$

where $b \in B$ and $F \in S^{\bullet} U$. In other words, the action of $d$ corresponds to the multiplication by the element $\sum_{i} x_{i} \cdot y_{i}$ in $R$ : it is immediate to observe the operator is indeed nilpotent, i.e. $d \circ d=0$ because the multiplying element $\sum_{i} x_{i} \cdot y_{i}$ is odd. It follows that $d$ makes $R$ into an actual complex $R:=K_{\text {e }}$ where the $\mathbb{Z}$-grading is induced by the (super) symmetric powers of $U$ as in (2.3) and the pair $\left(K_{\bullet}, d\right)$ is a differentially graded $(\mathrm{dg}) B$-algebra, which we call the (dual of the) Koszul complex associated with $A^{N}$, or Koszul complex of $A^{N}$ for short.

Before we compute the homology of the Koszul complex and for future use, we give a straightforward generalization of the Euler vector field and its action to a supercommutative setting. Let $f \in A\left[x_{1}, \ldots, x_{p} \mid \theta_{1}, \ldots, \theta_{q}\right]=S_{y m}{ }^{\bullet} V$ be a bi-homogeneous polynomial, i.e. homogeneous in the $x_{i}^{\prime}$ 's and in the $\theta_{j}$ 's. Then the map $f \mapsto \operatorname{deg} f$, which associates with $f$ its bi-homogeneous degree is well defined. Now, let $E$ be the Euler vector fields, i.e. the differential operator defined

$$
E:=\sum_{i=1}^{p} x_{i} \partial_{x_{i}}+\sum_{j=1}^{q} \theta_{q} \partial_{\theta_{q}}
$$

and acting on polynomials. Then one has the following easy lemma, which mimic the ordinary one for the commutative case. 
Lemma 2.1 Let $f\left(x_{1}, \ldots, x_{p} \mid \theta_{1}, \ldots, \theta_{q}\right) \in A\left[x_{1}, \ldots, x_{p} \mid \theta_{1}, \ldots, \theta_{q}\right]$ a bi-homogeneous polynomial and let $E:=\sum_{i=1}^{p} x_{i} \partial_{x_{i}}+\sum_{j=1}^{q} \theta_{q} \partial_{\theta_{q}}$ be the Euler vector field. Then

$$
(\operatorname{deg} f) f=E(f),
$$

where $\operatorname{deg} f$ is the degree of the bi-homogeneous polynomial $f$.

Proof Obvious, as if follows from the case of monomials.

Clearly, the above reduces to the ordinary result setting $\theta_{i}=0$ for any $i$. In particular this enters the proof of the homology of the Koszul complex, which-with reference to the above definition-is concentrated in degree $N$ as the following theorem shows.

Theorem 2.2 Let $(K ., d)$ the Koszul complex associate to $A^{N}$ for some A. Then the homology of $\left(R_{\bullet}, d\right)$ is concentrated in degree N. More in particular, we have

$$
H_{i}\left(\left(K_{\bullet}, d\right)\right) \cong\left\{\begin{array}{cc}
A \cdot y_{1} \ldots y_{N} & i=N \\
0 & \text { else }
\end{array}\right.
$$

Proof We construct a homotopy for $d: K_{i} \rightarrow K_{i+1}$. To this end, let us consider $h^{K}:=\sum_{i=1}^{N} \partial_{x_{i}} \partial_{y_{i}}: K_{i} \rightarrow K_{i-1}$. Without loss of generality, we can restrict to homogeneous elements $b \in B$ and $F \in S y m^{\bullet} U$ and we compute:

$$
\begin{aligned}
h^{K} d(b \cdot F) & =\sum_{j=1}^{N} \partial_{x_{j}} \cdot \partial_{y_{j}}\left(\sum_{i=1}^{N} x_{i} \cdot y_{i}(b \cdot F)\right)=\sum_{i, j=1}^{N} \partial_{x_{j}}\left(x_{i} b\right) \partial_{y_{j}}\left(y_{i} F\right) \\
& =\sum_{i, j=1}^{N}\left(\delta_{i j} b+x_{i} \cdot \partial_{x_{j}} f\right)\left(\delta_{i j} F-y_{i} \cdot \partial_{y_{j}} F\right) \\
& =\sum_{i}\left(\delta_{i i} b \cdot F-b \cdot\left(y_{i} \partial_{y_{i}} F\right)-\left(x_{i} \partial_{x_{i}} b\right) \cdot F-\left(x_{i} \cdot y_{i}\right)\left(\partial_{x_{i}} \partial_{y_{i}}\right) b \cdot F\right) \\
& =N b \cdot F-\operatorname{deg}(F) b \cdot F+\operatorname{deg}(b) b \cdot F-d h^{K}(b \cdot F) .
\end{aligned}
$$

This leads to

$$
h^{K} d+d h^{K}=N-\operatorname{deg}(F)+\operatorname{deg}(b) .
$$

Since $\operatorname{deg}(F) \leq N$ we have that $N-\operatorname{deg}(F) \geq 0$, so that if $\operatorname{deg}(b)>0$ the sum above is never zero, and for any such pair $(\operatorname{deg}(b), \operatorname{deg}(F))$ with $\operatorname{deg}(b)>0$ and $0 \leq \operatorname{deg}(F) \leq N$ we can define a homotopy operator for $d$ as

$$
h_{\operatorname{deg}(b), \operatorname{deg}(F)}^{K}:=\frac{h^{K}}{N-\operatorname{deg}(F)+\operatorname{deg}(b)}: K_{i} \rightarrow K_{i-1} .
$$

The only instance in which the homotopy fails is when $\operatorname{deg}(F)=N$ and $\operatorname{deg}(b)=0$ : the generator of the corresponding module is $A \cdot y_{1} \ldots y_{n}$ which is clearly in the kernel of $d$.

The geometrical upshot of this important theorem is that the determinant or canonical module $\wedge^{N} A^{N}$ related to $A^{N}$ emerges as the (co)homology of the Koszul complex of $A^{N}$. We will make use of this result later on in the paper. 


\section{The Super Koszul Complex and its homology}

In this section, we define a super analog of the ordinary, i.e. commutative, Koszul complex, and we shall see that, as in the ordinary commutative setting, also in the supercommutative setting the Koszul complex yields a resolution of $A$ as a $R$-module, with $R$ given by (2.1).

For future convenience, given basis of $\left\{x_{1}, \ldots, x_{p} \mid \theta_{1}, \ldots, \theta_{q}\right\}$ of $V=A^{p \mid q}$, we introduce the basis $\left\{\ell_{1}, \ldots, \ell_{q} \mid \chi_{i}, \ldots, \chi_{p}\right\}$ of $\Pi V$, where the have set $\ell_{j}:=\pi \theta_{j}$ and $\chi_{i}:=\pi x_{i}$, so that $\left|\ell_{j}\right|=0$ and $\left|\chi_{i}\right|=1$ for any $i=1, \ldots, p$ and $j=1, \ldots, q$. Notice that if $V$ has dimension $p \mid q$, then $\Pi V$ has dimension $q \mid p$.

As a warm-up, with reference to the previous section, let us consider the following composition of maps:

$$
R \otimes S y m^{2} \Pi V \stackrel{\delta_{2}}{\longrightarrow} R \otimes S y m^{1} \Pi V \stackrel{\delta_{1}}{\longrightarrow} R
$$

where $\delta_{1}: R \otimes \Pi V \rightarrow R$ is given by the composition of the presentation $p: R \otimes \Pi V \rightarrow I_{1}$ of the ideal $I_{1} \subset R$ with the immersion $i: I_{1} \hookrightarrow R$, so that $\delta_{1}:=i \circ p: R \otimes \Pi V \rightarrow R$ acts as already defined in equation (2.4), which employing the $\left\{\ell_{j} \mid \chi_{i}\right\}$-notation introduced above now reads:

$$
\begin{aligned}
& 1 \otimes \ell_{j} \stackrel{\delta_{1}}{\longmapsto} \theta_{j}, \\
& 1 \otimes \chi_{i} \stackrel{\delta_{1}}{\longmapsto} x_{i} .
\end{aligned}
$$

The map $\delta_{2}: R \otimes S y m^{2} \Pi V \rightarrow R \otimes \Pi V$ is defined on a basis of $R \otimes S y m^{2} \Pi V$ as follows:

$$
\begin{aligned}
1 \otimes\left(\ell_{i} \odot \ell_{j}\right) & =1 \otimes\left(\pi \theta_{i} \odot \pi \theta_{j}\right) \stackrel{\delta_{2}}{\longmapsto} \theta_{i} \otimes \pi \theta_{j}+\theta_{j} \otimes \pi \theta_{i}=\theta_{i} \otimes \ell_{j}+\theta_{j} \otimes \ell_{i}, \\
1 \otimes\left(\ell_{i} \odot \chi_{k}\right) & =1 \otimes\left(\pi \theta_{i} \odot \pi x_{k}\right) \stackrel{\delta_{2}}{\longmapsto} \theta_{i} \otimes \pi x_{k}+x_{k} \otimes \pi \theta_{i}=\theta_{i} \otimes \chi_{k}+x_{k} \otimes \ell_{j} \\
1 \otimes\left(\chi_{k} \odot \chi_{l}\right) & =1 \otimes\left(\pi x_{k} \odot \pi x_{l}\right) \stackrel{\delta_{2}}{\longmapsto} x_{k} \otimes \pi x_{l}-x_{l} \otimes \pi x_{k}=x_{k} \otimes \chi_{l}-x_{l} \otimes \chi_{k} .
\end{aligned}
$$

It is straightforward to observe that these elements are in kernel of the map $\delta_{1}$, so that one has that $\delta_{2} \circ \delta_{1}=0$. This is not by accident and indeed the above construction can be made general.

Having already defined $R:=\bigoplus_{k \geq 0} S y m^{k} V$, we further introduce

$$
R^{\pi}:=\bigoplus_{k \geq 0} R_{k}^{\pi} \quad \text { with } \quad R_{k}^{\pi}:=S y m^{k} \Pi V,
$$

and in turn, we define the tensor product of $R$ and $R^{\pi}$ over $A$ :

$$
\mathcal{K}_{.}:=\bigoplus_{k \geq 0} \mathcal{K}_{-k}=R \otimes_{A} \bigoplus_{k \geq 0} R_{k}^{\pi}=R \otimes_{A} R^{\pi} .
$$

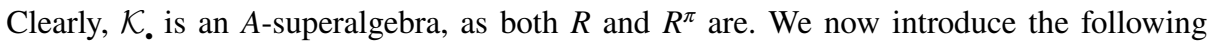
$A$-superalgebra homomorphism: 


$$
\begin{aligned}
\delta: \mathcal{K}_{\bullet} & =R \otimes_{A} R^{\pi} \longrightarrow \mathcal{K}_{\bullet}=R \otimes_{A} R^{\pi} \\
r & \otimes r^{\pi} \longmapsto \delta\left(r \otimes r^{\pi}\right):=\left(\sum_{i=1}^{p} x_{i} \otimes \partial_{\chi_{i}}+\sum_{j=1}^{q} \theta_{j} \otimes \partial_{\ell_{j}}\right)\left(r \otimes r^{\pi}\right),
\end{aligned}
$$

where $r \in R$ and $r^{\pi} \in R^{\pi}$. It is immediate to observe the following facts:

1. with respect to the supercommutative structure of $\mathcal{K}_{\bullet}$, that is with respect to the $\mathbb{Z}_{2}$ -gradation, $\delta$ is odd, i.e. $|\delta|=1$;

2. with respect to the $\mathbb{Z}$-gradation of $\mathcal{K}$. as an $R$-module, $\delta$ is homogeneous of degree -1 .

3. $\delta$ acts as a derivation only on the factor $R^{\pi}$. It follows that $\delta$ is $R$-linear on $\mathcal{K}_{\bullet}=R \otimes_{A} R^{\pi}$ endowed with the structure of an $R$-module (actually, $R$-algebra) induces by its factor $R$.

We have the following theorem.

Theorem 3.1 The pair $\left(\mathcal{K}_{\bullet}, \delta\right)$ defines a differentially graded $(d g) R$-algebra.

Proof We have already observed that $\delta$ is $R$-linear. We need to prove that $\delta^{2}:=\delta \circ \delta=0$. We observe that $\mathcal{K}_{\text {. }}$ is generated as an $R$-algebra by $\operatorname{Sym}^{1} \Pi V \cong \Pi V$, which is $\mathcal{K}_{-1}$ upon tensoring with $R$, i.e. looking at $\mathcal{K}$. as an $R$-algebra. It follows that it is enough to verify that $\delta^{2}\left(1 \otimes \ell_{j}\right)=0$ and $\delta^{2}\left(1 \otimes \chi_{i}\right)$ for any $j=1, \ldots, q$ and $i=1, \ldots, p$, where $\left\{1 \otimes \ell_{j} \mid 1 \otimes \chi_{i}\right\}$ are the generators of $\Pi V$, and then work by induction on the $\mathbb{Z}$-degree of $\mathcal{K}$. Obviously, one has

$$
\begin{aligned}
& \delta^{2}\left(1 \otimes \ell_{j}\right)=\delta\left(x_{j} \otimes 1\right)=0, \\
& \delta^{2}\left(1 \otimes \chi_{j}\right)=\delta\left(\theta_{j} \otimes 1\right)=0,
\end{aligned}
$$

which settle the case $\mathcal{K}_{-1}$. Let us now assume that $s \in \mathcal{K}_{-1}$ and $t \in \mathcal{K}_{-k}$ for $k \geq 1$ satisfying $\delta^{2}(t)=0$ by induction hypothesis. By Leibniz rule, for an element $s \cdot t \in \mathcal{K}_{-k-1}$, one has

$$
\begin{aligned}
\delta^{2}(s \cdot t) & =\delta^{2}(s) t+(-1)^{|\delta||\delta(s)|} \delta(s) \delta(t)+(-1)^{|\delta||s|} \delta(s) \delta(t)+(-1)^{2|\delta||s|} s \delta^{2}(t) \\
& =(-1)^{|s|+1} \delta(s) \delta(t)+(-1)^{|s|} \delta(s) \delta(t)+s \delta^{2}(t) \\
& =s \delta^{2}(t)=0
\end{aligned}
$$

by induction hypothesis, recalling that $|\delta|=1$ so that, in $\operatorname{particular}|\delta(s)|=s+1$.

The previous theorem justifies the following definition.

Definition 3.2 (Super Koszul Complex) Given any free $A$-module $V=A^{p \mid q}$ for any superalgebra $A$, we call the pair $\left(\mathcal{K}_{\bullet}, \delta\right)$ the super Koszul complex associated with $V$ : 


\section{$\cdots \stackrel{\delta}{\longrightarrow} R \otimes S y m^{k} \Pi V \stackrel{\delta}{\longrightarrow} \cdots \stackrel{\delta}{\longrightarrow} R \otimes S y m^{2} \Pi V \stackrel{\delta}{\longrightarrow} R \otimes \Pi V \stackrel{\delta}{\longrightarrow} R \longrightarrow 0$.}

We are interested to studying the homology of the super Koszul complex. The crucial result in this direction is the construction of a homotopy operator $h_{\bullet}^{\mathcal{K}}: \mathcal{K}_{\bullet} \rightarrow \mathcal{K}$. for the differential of the super Koszul complex, with $h_{i}^{\mathcal{K}}: \mathcal{K}_{-i} \rightarrow \mathcal{K}_{-i-1}$ such that $h_{i+1}^{\mathcal{K}} \circ \delta_{i}+\delta_{i-1} \circ h_{i}^{\mathcal{K}}=i d_{\mathcal{K}_{i}}$. We organize the construction of the homotopy in two consequential lemmas, whose proofs rely on Lemma 2.1 above.

Lemma 3.3 Let $\left(\mathcal{K}_{.}, \delta\right)$ be the super Koszul complex associated with some $V=A^{p \mid q}$ with basis as above. Then the operator $\varepsilon: \mathcal{K}_{\bullet} \rightarrow \mathcal{K}_{\bullet}$ defined as

$$
\varepsilon:=\sum_{i=1}^{p} \partial_{x_{i}} \otimes \chi_{i}+\sum_{j=1}^{q} \partial_{\theta_{j}} \otimes \ell_{j}
$$

is such that

$$
\delta \circ \varepsilon+\varepsilon \circ \delta=E \otimes i d_{R^{\pi}}+i d_{R} \otimes E^{\pi},
$$

for $E$ the Euler vector field acting on $R=S^{\bullet} m^{\bullet} V$ and $E^{\pi}$ the Euler vector field acting on $R^{\pi}=\operatorname{Sym} \cdot \Pi V$.

Proof We first notice that notice that $\varepsilon$ defined as above raises the degree by one in $\mathcal{K}$., i.e. $\varepsilon: \mathcal{K}_{i} \rightarrow \mathcal{K}_{i+1}$ since it multiplies by the elements $\chi_{i}$ and $\ell_{j}$. Also, it is odd-likewise $\delta$ and it is symmetric to $\delta$, in that it is a derivation on the first factor of $R \otimes_{A} R^{\pi}=\mathcal{K}_{\bullet}$, while $\delta$ was a derivation on the second factor of $R \otimes_{A} R^{\pi}=\mathcal{K}$.

Let us now compute the commutator $[\delta, \varepsilon]=\delta \circ \varepsilon+\varepsilon \circ \delta$. By applying the definitions, one has that

$$
\begin{aligned}
& \delta \circ \varepsilon=\sum_{i=1}^{p} x_{i} \partial_{x_{i}} \otimes i d_{R^{\pi}}+\sum_{j=1}^{q} \theta_{j} \partial_{\theta_{j}} \otimes i d_{R^{\pi}}, \\
& \varepsilon \circ \delta=i d_{R} \otimes \sum_{i=1}^{p} \chi_{i} \partial_{\chi_{i}}+i d_{R} \otimes \sum_{j=1}^{q} \ell_{j} \partial_{\ell_{j}},
\end{aligned}
$$

so that one has

$$
[\delta, \varepsilon]=\left(\sum_{i=1}^{p} x_{i} \partial_{x_{i}}+\sum_{j=1}^{q} \theta_{j} \partial_{\theta_{j}}\right) \otimes i d_{R^{\pi}}+i d_{R} \otimes\left(\sum_{i=1}^{p} \chi_{i} \partial_{\chi_{i}}+\sum_{j=1}^{q} \ell_{j} \partial_{\ell_{j}}\right),
$$

which in turn can be rewritten as

$$
[\delta, \varepsilon]=E \otimes i d_{R^{\pi}}+i d_{R} \otimes E^{\pi},
$$

where $E$ and $E^{\pi}$ are the Euler vector fields acting on $R$ and $R^{\pi}$ respectively.

Upon a suitable normalization, the operator $\varepsilon$ defined in the previous lemma allows us to write the homotopy for the Koszul complex, as we show in the following. 
Lemma 3.4 (Homotopy of $\delta$ ) Let $\left(\mathcal{K}_{.}, \delta\right)$ be the super Koszul complex associated with some $V=A^{p \mid q}$ with basis as above and let $\varepsilon: \mathcal{K}_{\bullet} \rightarrow \mathcal{K}$. be defined as in Lemma 3.3. Then the operator $\varepsilon_{k, i}:=\frac{1}{k+i} \varepsilon$ with

$$
\varepsilon:=\sum_{i=1}^{p} \partial_{x_{i}} \otimes \chi_{i}+\sum_{j=1}^{q} \partial_{\theta_{j}} \otimes \ell_{j}
$$

is such that

$$
\delta \circ \varepsilon_{k, i}+\varepsilon_{k+1, i-1} \circ \delta=i d_{R} \otimes i d_{R^{\pi}} .
$$

In particular, $h_{\bullet}^{\mathcal{K}}:=\bigoplus_{k, i \geq 0} \varepsilon_{k, i}: \mathcal{K}_{\bullet} \rightarrow \mathcal{K}_{\bullet}$ with

$$
h_{i}^{\mathcal{K}}:=\bigoplus_{k \geq 0} \varepsilon_{k, i}: \mathcal{K}_{-i}=R \otimes_{A} R_{i}^{\pi} \longrightarrow \mathcal{K}_{-i-1}=R \otimes_{A} R_{i+1}^{\pi}
$$

defines a homotopy for the differential of the super Koszul complex.

Proof Notice that $\varepsilon_{k, i}: R_{k} \otimes R_{i}^{\pi} \rightarrow R_{k-1} \otimes R_{i+1}^{\pi}$ is just a normalization of $\varepsilon$. Further, notice that, symmetrically, the Koszul differential $\delta$ acts on the homogeneous factors of $\mathcal{K} .=R \otimes_{A} R^{\pi}$ as $\delta: R_{k} \otimes R_{i}^{\pi} \rightarrow R_{k+1} \otimes R_{i-1}^{\pi}$. We can thus consider the following diagram

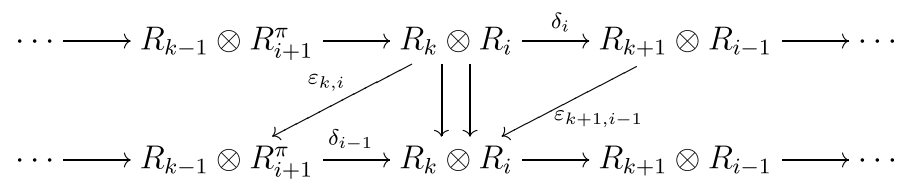

We first observe that, as for the normalization, one has

$$
\varepsilon_{k, i}=\frac{1}{k+i} \varepsilon=\varepsilon_{k+1, i-1} .
$$

Therefore, it follows from the computation of the commutator in the previous lemma 3.3 that we can set

$$
\delta \circ \varepsilon_{k, i}+\varepsilon_{k+1, i-1} \circ \delta=\frac{1}{k+i}[\delta, \varepsilon]=\frac{1}{k+i}\left(E \otimes i d_{R^{\pi}}+i d_{R} \otimes E^{\pi}\right) .
$$

Applying lemma 2.1, since the degrees corresponds to the powers $k$ and $i$ for $R_{k}$ and $R_{i}^{\pi}$ respectively, one gets,

$$
\frac{1}{k+i} E \otimes i d_{R^{\pi}}+i d_{R} \otimes E^{\pi}=\frac{k+i}{k+i} i d_{R} \otimes i d_{R^{\pi}}=i d_{R} \otimes i d_{R^{\pi}},
$$

establishing the first part of the lemma. For the second part, it is enough to observe that the (-1)-th terms of the super Koszul complex can be written as $\mathcal{K}_{-i}=R \otimes_{A} \operatorname{Sym}^{i} \Pi V=\left(\bigoplus_{k \geq 0} R_{k}\right) \otimes_{A} R_{i}^{\pi}$. 
The previous lemma allows to compute the homology of the super Koszul complex, the main result of the present section.

Theorem 3.5 (Homology of $\mathcal{K}_{\bullet}$ ) The homology of the super Koszul complex $\left(\mathcal{K}_{\bullet}, \delta\right)$ is given by

$$
H_{i}\left(\left(\mathcal{K}_{\bullet}, \delta\right)\right) \cong\left\{\begin{array}{cc}
A & i=0 \\
0 & i \neq 0
\end{array}\right.
$$

In particular $\left(\mathcal{K}_{.}, \delta\right)$ is an exact resolution of $A$ endowed with the structure of $R$-module.

Proof It is enough to observe that the homotopy operator $h_{\bullet}^{\mathcal{K}}: \mathcal{K}_{\bullet} \rightarrow \mathcal{K}_{\text {. introduced in the }}$ previous lemma 3.4 is defined for $k+i>0$. It follows that, when $h_{\bullet}^{\mathcal{K}}$ is defined, i.e. for any $k+i \geq 0$, we have

$$
H_{i}\left(\mathcal{K}_{\bullet}\right)_{k}=0 \text {, }
$$

where $H_{i}\left(\mathcal{K}_{\bullet}\right)_{k}$ is the $R$-degree $k$-component of $H_{i}\left(\mathcal{K}_{\bullet}\right)$, with the structure of $\mathbb{Z}$-graded $R$-module inherited by that of $\mathcal{K}$. The only homology group of $\mathcal{K}$. which is not annihilated by the homotopy corresponds to the choice $i=0=k$, i.e. $H_{0}\left(\mathcal{K}_{\bullet}\right)_{0}$. In this case, the complex reads

$$
\cdots \longrightarrow 0 \longrightarrow 0 \longrightarrow A \longrightarrow 0
$$

so that $H_{0}(\mathcal{K} .)_{0}=A$, and the result follows.

Remark 3.6 Before we pass to the next section, a remark is now in order. Indeed the ring $R$ entering the construction of the super Koszul complex is a ring of polynomials in variables that are by no means a regular sequence in general, due to the presence of odd variables. This is the reason why the usual induction proof - that can be found for example in [7] — of the analog of Theorem 3.5 in the commutative case does not extend to the supercommutative case, thus leading us to make use of the homotopy previously constructed in Lemmas 3.3 and 3.4. It is worth noticing by the way that the above computation of the homology breakdown in the case the characteristic of $A$ is different than zero, leading to an interesting and richer scenario as the following example shows.

Example 3.7 (Homology of Super Koszul Complex in $\operatorname{char}(A)=p$ ) Let us set $\operatorname{char}(A)=3$, for example $A:=\mathbb{Z}_{3}$ and let us consider two variable, $x$ even and $\theta$ odd and set, as above, $\chi=\pi x$ and $\ell=\pi \theta$. We therefore have $V=\mathbb{Z}_{3} x \oplus \mathbb{Z}_{3} \theta=\mathbb{Z}_{3}^{1 \mid 1}$ and hence

$$
R:=\operatorname{Sym}^{\bullet} V=\mathbb{Z}_{3}[x \mid \theta], \quad R^{\pi}:=\operatorname{Sym} \cdot \Pi V=\mathbb{Z}_{3}[\ell \mid \chi] .
$$

This leads to consider the super Koszul complex given by $R \otimes_{\mathbb{Z}} R^{\pi}=\mathbb{Z}_{3}[x, \ell \mid \chi, \theta]$, having differential defined by $\delta=x \partial_{\chi}+\theta \partial_{\ell}$. In the notation $\left(\mathcal{K}_{\bullet}, \delta\right)$, we have that

$$
\ldots \longrightarrow \mathcal{K}_{-3} \stackrel{\delta}{\longrightarrow} \mathcal{K}_{-2} \stackrel{\delta}{\longrightarrow} \mathcal{K}_{-1} \stackrel{\delta}{\longrightarrow} \mathcal{K}_{0} \longrightarrow 0
$$


corresponds to

$$
\ldots \longrightarrow R \cdot \ell^{3} \oplus R \cdot \ell^{2} \chi \stackrel{\delta}{\longrightarrow} R \cdot \ell^{2} \oplus R \cdot \ell \chi \stackrel{\delta}{\longrightarrow} R \cdot \ell \oplus R \cdot \chi \stackrel{\delta}{\longrightarrow} R \longrightarrow 0
$$

Now consider the element $\theta \ell^{2} \in \mathcal{K}_{-2}$ : it is straightforward to check that it is a cycle, i.e. $\delta\left(\theta \ell^{2}\right)=0$. On the other hand, for any element $\tau \in \mathcal{K}_{-3}$, with $\tau:=f(x \mid \theta) \ell^{3}+g(x \mid \theta) \ell^{2} \chi \in \mathcal{K}_{-3}$, one has that $\delta(\tau)=2 \theta g(x \mid \theta) \ell \chi+x g(x \mid \theta) \ell^{2}$, so that in particular $\theta \ell^{2}$ is not a boundary, i.e. there is no $\tau \in \mathcal{K}_{-3}$ such that $\theta \ell^{2} \neq \delta(\tau)$ and therefore $\left[\theta \ell^{3}\right] \in H_{2}\left(\left(\mathcal{K}_{\bullet}, \delta\right)\right) \neq 0$.

\section{The dual of the Super Koszul Complex and its homology}

Given the super Koszul complex $\left(\mathcal{K}_{.}, \delta\right)$ associated with $V=A^{p \mid q}$, we can define its dual via the functor $\operatorname{Hom}_{R}(-, R)$, for $R=\operatorname{Sym}^{\bullet} V$ as above. Doing so, one gets the pair $\left(\mathcal{K}_{\bullet}^{*}, \delta^{*}\right):=\left(\operatorname{Hom}_{R}\left(\mathcal{K}_{\bullet}, R\right), \operatorname{Hom}_{R}(\delta, R)\right)$. Defining

$$
R_{k}^{\pi *}:=\bigoplus_{k \geq 0} R_{i}^{\pi *} \quad \text { with } \quad R_{k}^{\pi *}:=S y m^{k} \Pi V^{*}
$$

the complex $\mathcal{K}^{*}$ is thus given by

$$
\mathcal{K}^{*}=\bigoplus_{k \geq 0} \mathcal{K}_{k}^{*}=R \otimes_{A} \bigoplus_{k \geq 0} R_{k}^{\pi *}=R \otimes_{A} R^{\pi *}
$$

Notice that $R^{\pi *}$ is a $A$-superalgebra generated by the elements $\left\{\partial_{\ell_{1}}, \ldots, \partial_{\ell_{q}} \mid \partial_{\chi_{1}}, \ldots, \partial_{\chi_{p}}\right\}$ for $\ell_{i}:=\pi \theta_{i}$ and $\chi_{j}:=\pi x_{j}$ for $i=1, \ldots, q$ and $j=1, \ldots, p$.

The operator $\delta^{*}: \mathcal{K}^{*} \rightarrow \mathcal{K}_{\text {. }}^{*}$ is formally identical to $\delta: \mathcal{K}_{\bullet} \rightarrow \mathcal{K}_{\bullet}$, the differential of the super Koszul complex introduced above, but what is crucial to note is that $\delta^{*}$ should now be seen as the multiplication operator by the odd element $\sum_{j} x_{j} \otimes \partial_{\chi_{j}}+\sum_{i} \theta_{i} \otimes \partial_{\ell_{i}}$ in the superalgebra $R \otimes_{A} R^{\pi *}$. Once this is acknowledged, we still write $\delta^{*}$ as

$$
\delta^{*}:=\sum_{j=1}^{p} x_{j} \otimes \partial_{\chi_{j}}+\sum_{i=1}^{q} \theta_{i} \otimes \partial_{\ell_{i}} .
$$

Further, note that since $\delta^{*}$ acts as the multiplication by an odd element, it is automatically nilpotent, i.e. $\delta^{*} \circ \delta^{*}=0$ : this justifies the following definition.

Definition 4.1 (Dual of the Super Koszul Complex) Given any free $A$-module $V=A^{p \mid q}$ for any superalgebra $A$, we call the pair $\left(\mathcal{K}_{\bullet}^{*}, \delta^{*}\right)$ the dual of the super Koszul complex associated with $V$.

$$
0 \longrightarrow R \stackrel{\delta^{*}}{\longrightarrow} R \otimes \Pi V^{*} \stackrel{\delta^{*}}{\longrightarrow} R \otimes S y m^{2} \Pi V^{*} \stackrel{\delta^{*}}{\longrightarrow} \ldots \stackrel{\delta^{*}}{\longrightarrow} R \otimes S y m^{k} \Pi V^{*} \stackrel{\delta^{*}}{\longrightarrow} \ldots
$$


Before we go on and study the homology of this complex, let us briefly discuss the functoriality of the above construction, as to understand the properties of the functor $V \mapsto \mathcal{K}^{*}$.

Given two $A$-supermodules $V$ and $W$, applying the functor $S y m^{*}(-): \mathbf{S M o d}_{A} \rightarrow \mathbf{S A l g}_{A}$, one gets the $A$-superalgebras $R^{V}:=\bigoplus_{i \geq 0} \operatorname{Sym}^{i} V$, and $R^{W}:=\bigoplus_{i \geq 0} \operatorname{Sym}^{i} W$. As for the arrows, given a homomorphism $f: V \rightarrow W$ of $A$-supermodules, the action of the functor yields a supercommutative $A$-algebra morphism:

$$
f \longmapsto \operatorname{Sym}^{\bullet}(f): R^{V} \longrightarrow R^{W} .
$$

Likewise, considering a second homomorphism $f^{\pi}: \Pi V \rightarrow \Pi W$, upon applying $\operatorname{Sym}^{\bullet}(-)$ to the direct sum $f \oplus f^{\pi}: V \oplus \Pi V \rightarrow W \oplus \Pi W$, one gets

$$
f \oplus f^{\pi} \longmapsto \operatorname{Sym}^{\bullet}\left(f \oplus f^{\pi}\right): R^{V} \otimes_{A} R^{V \pi} \longrightarrow R^{W} \otimes_{A} R^{W \pi},
$$

which corresponds to a morphism between the super Koszul complex associated with $V$ we call it $\mathcal{K}_{\text {. }}^{V}$-and the super Koszul complex associated with $W$-we call it $\mathcal{K}_{\text {. }}^{W}$ :

$$
f_{\bullet}^{V W}:=\operatorname{Sym}^{\bullet}\left(f \oplus f^{\pi}\right): \mathcal{K}_{\bullet}^{V} \longrightarrow \mathcal{K}_{\bullet}^{W} .
$$

Let us apply the functor $\operatorname{Hom}_{R^{W}}\left(-, R^{W}\right)$ : one has the following commutative triangle

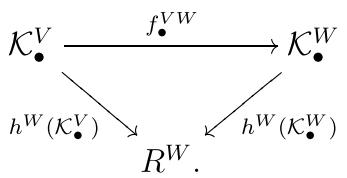

The action on the functor on the morphisms gives the following map

$$
\begin{aligned}
& \left(f_{\bullet}^{V W}\right)^{*}: \operatorname{Hom}_{R^{W}}\left(\mathcal{K}_{\bullet}^{W}, R^{W}\right) \longrightarrow \operatorname{Hom}_{R^{W}}\left(\mathcal{K}_{\bullet}^{V}, R^{W}\right) \\
& h^{W}\left(\mathcal{K}_{\bullet}^{W}\right) \longmapsto h^{W}\left(\mathcal{K}_{\bullet}^{V}\right):=h^{W}\left(\mathcal{K}_{\bullet}^{W}\right) \circ f_{\bullet}^{V W},
\end{aligned}
$$

where we have defined $\left(f^{V W}\right)^{*}:=\operatorname{Hom}_{R^{W}}\left(f^{V W}, R^{W}\right)$. It follows that the functor $V \mapsto \mathcal{K}^{*}$. is not strictly a contravariant functor. Indeed observing that

$$
\operatorname{Hom}_{R^{W}}\left(\mathcal{K}_{\bullet}^{V}, R^{W}\right)=\operatorname{Hom}_{R^{V}}\left(\mathcal{K}_{\bullet}^{V}, R^{V}\right) \bigotimes_{R^{V}} \otimes R^{W}=\mathcal{K}_{\bullet}^{V^{*}} \bigotimes_{R^{V}} R^{W},
$$

the previous (4.9) reads

$$
\left(f_{\bullet}^{V W}\right)^{*}: \mathcal{K}_{\bullet}^{W *} \longrightarrow \mathcal{K}_{\bullet}^{V *} \otimes_{R^{V}} R^{W}
$$

Nonetheless, let us assume that $f \in \operatorname{Aut}(V)$ - for example, $f$ is a change of basis. Then, in this case, we have a map $\left(f_{.}^{V}\right)^{*}: \mathcal{K}_{\text {. }}^{V^{*}} \longrightarrow \mathcal{K}_{\text {. }}^{V *}$. More precisely one finds

$$
\left(f^{V}\right)^{*}=\operatorname{Sym}^{*}\left(f \oplus\left(f^{\pi}\right)^{t}\right): \mathcal{K}_{\bullet}^{V *} \longrightarrow \mathcal{K}_{\bullet}^{V^{*}} .
$$

and one gets a contravariant functor $V \mapsto \mathcal{K}_{\text {. }}^{V *}$ with $\left(f_{\bullet}^{V} \circ g_{\bullet}^{V}\right)^{*}=\left(g_{\bullet}^{V}\right)^{*} \circ\left(f_{\bullet}^{V}\right)^{*}$, as can be readily checked. Finally, notice that because of their particular form, the homological operators 
$\delta$ and $\delta^{*}$, differential of the super Kozsul complex and its dual respectively, are invariant under change of basis, i.e. automorphisms of $V$.

As done in the previous section for the super Koszul complex $\left(\mathcal{K}_{\bullet}, \delta\right)$, we are now interested into computing the homology of the dual of the super Koszul complex $\left(\mathcal{K}_{.}^{*}, \delta^{*}\right)$.

To this end, recalling that the operator $\delta^{*}=\sum_{i} x_{i} \otimes \partial_{\chi_{i}}+\sum_{j} \theta_{j} \otimes \partial_{\ell_{j}}$ is now looked as a multiplication operator in the algebra $\mathcal{K}_{.}^{*}=R \otimes R^{\pi *}$, one immediately gets an inclusion of ideals.

Indeed, clearly, $\left(\delta^{*}\right)^{2}=0$, so the element corresponding to

$$
\delta^{*}:=\sum_{j=1}^{p} x_{j} \otimes \partial_{\chi_{j}}+\sum_{i=1}^{q} \theta_{i} \otimes \partial_{\ell_{i}} \in V \otimes \Pi V^{*}
$$

is in the kernel of $\delta^{*}$ seen as the multiplication operator, i.e. $\delta^{*} \subseteq \operatorname{ker}\left(\delta^{*}\right)$.

On the other hand it is immediate to observe that also the element

$$
\mathcal{D}:=\theta_{1} \ldots \theta_{q} \otimes \partial_{\chi_{1}} \ldots \partial_{\chi_{p}} \in S y m^{q} V \otimes \operatorname{Sym}^{p} \Pi V^{*}
$$

is in the kernel of $\delta^{*}$, since both the factor in $S y m^{q} V$ and the factor in $S y m^{p} \Pi V^{*}$ are completely antisymmetric and when they get multiplied by another odd term coming from $\delta$ they yield zero, i.e. $\mathcal{D} \in \operatorname{ker}\left(\delta^{*}\right)$. We therefore have the following inclusion of ideals

$$
\left(\delta^{*}, \mathcal{D}\right) \subseteq \operatorname{Ker}\left(\delta^{*}\right),
$$

where, as it is customary, $\left(\delta^{*}, \mathcal{D}\right)$ denotes the ideal generated by the elements $\delta^{*}$ and $\mathcal{D}$. We now prove that such an inclusion is indeed an equality.

Lemma 4.2 Let $\left(\mathcal{K}^{*}, \delta^{*}\right)$ the dual of the super Koszul complex associated with $V=A^{p \mid q}$ for some A. Then $\operatorname{ker}\left(\delta^{*}\right)=\left(\delta^{*}, \mathcal{D}\right)$. In particular

$$
\operatorname{ker}\left(\delta^{*}\right) / \operatorname{im}\left(\delta^{*}\right)=(\mathcal{D}) .
$$

Proof The proof of this lemma relies on the ordinary Koszul complex construction of the first section. Let us start simplifying the notation. It can be observed that, by definition

$$
R \otimes_{A} R^{\pi *}=A\left[x_{1}, \ldots x_{p}, \partial_{\ell_{1}}, \ldots, \partial_{\ell_{q}} \mid \partial_{\chi_{1}}, \ldots, \partial_{\chi_{p}}, \theta_{1}, \ldots, \theta_{q}\right]
$$

where the even and odd generators have been grouped together. Posing $N:=p+q$ we define

$$
\begin{aligned}
& \left(u_{1}, \ldots, u_{N}\right):=\left(x_{1}, \ldots x_{p}, \partial_{\ell_{1}}, \ldots, \partial_{\ell_{q}}\right), \\
& \left(\psi_{1}, \ldots, \psi_{N}\right):=\left(\partial_{\chi_{1}}, \ldots, \partial_{\chi_{p}}, \theta_{1}, \ldots, \theta_{q}\right),
\end{aligned}
$$

so that, upon this redefinition, $\delta^{*}$ and $\mathcal{D}$ read

$$
\begin{aligned}
& \delta^{*}:=\sum_{i=1}^{N} u_{i} \psi_{i} \in A\left[u_{1}, \ldots, u_{N}, \psi_{1}, \ldots, \psi_{N}\right], \\
& \mathcal{D}=\prod_{j=1}^{N} \psi_{i} \in A\left[u_{1}, \ldots, u_{N}, \psi_{1}, \ldots, \psi_{N}\right] .
\end{aligned}
$$


as elements of the ring $A\left[u_{1}, \ldots, u_{N}, \psi_{1}, \ldots, \psi_{N}\right]$. Let us set $B:=A\left[u_{1}, \ldots, u_{N}\right]$ so that one has $A\left[u_{1}, \ldots, u_{N}, \psi_{1}, \ldots, \psi_{N}\right]=B\left[\psi_{1}, \ldots, \psi_{N}\right]$. By anticommutativity of the $\psi_{i}$ 's one has

$$
B\left[\psi_{1}, \ldots, \psi_{N}\right]=B \oplus \sum_{i=1}^{N} B \cdot \psi_{i} \oplus \ldots \oplus B \cdot\left(\psi_{1} \ldots \psi_{N}\right),
$$

but this is nothing but the ordinary (dual of the) Koszul complex $K$. introduced above in (2.3) and the result follows from theorem 2.2, that proved that the homology is generated over $A$ by the element $\mathcal{D}=\psi_{1} \ldots \psi_{N}$.

Recalling that we have proved in the previous section that the super Koszul complex $\mathcal{K}$. is an exact resolution of $A$ seen as $R$-module, and that $\operatorname{Ext}_{R}^{i}(A, R):=H^{i}\left(\operatorname{Hom}_{R}\left(\mathcal{K}_{\bullet}, R\right)\right)=H^{i}\left(\left(\mathcal{K}_{\bullet}^{*}, \delta^{*}\right)\right)$, we can finally compute the homology of the dual of super Koszul complex.

Theorem 4.3 (Homology of $\mathcal{K}_{.}^{*}$ ) The homology of the dual of the Koszul supercomplex $\left(\mathcal{K}_{.}^{*}, \delta^{*}\right)$ is given by

$$
\operatorname{Ext}_{R}^{i}(A, R)=\left\{\begin{array}{cl}
\Pi^{p+q} A & i=p \\
0 & \text { else. }
\end{array}\right.
$$

In particular, in the above notation, a generator is given by the element $\theta_{1} \ldots \theta_{q} \otimes \partial_{\chi_{1}} \ldots \partial_{\chi_{p}} \in \mathcal{K}_{p}^{*}$.

Proof Recovering the original notation, one has the following correspondence

$$
\psi_{1} \ldots \psi_{N}=\theta_{1} \ldots \theta_{q} \otimes \partial_{\chi_{1}} \ldots \partial_{\chi_{p}} \in \mathcal{K}_{\cdot}^{*}=R \otimes_{A} R^{\pi *}
$$

More precisely one sees that $\theta_{1} \ldots \theta_{q} \otimes \partial_{\chi_{1}} \ldots \partial_{\chi_{p}} \in S y m^{q} V \otimes_{A} S y m^{p} \Pi V^{*}$, which implies that $\theta_{1} \ldots \theta_{q} \otimes \partial_{\chi_{1}} \ldots \partial_{\chi_{p}} \in \mathcal{K}_{p}^{*}$. By lemma 4.2 above, it generates the cohomology of $\mathcal{K}_{.}^{*}$ as a $A$-supermodule and its parity depends on the sum $N=p+q$, so that the conclusion follows.

\section{Super Koszul Complex and the Berezinian}

We now aim at interpreting the main result of the previous section, and we show that $\operatorname{Ext}_{R}^{p}(A, R)$ transforms exactly as the (inverse of the) Berezinian module of $V$. More precisely, we prove the following theorem.

Theorem 5.1 Let $\phi \in A u t_{A}(V)$ be an automorphism of the free A-supermodule $V$. Then the induced automorphism $\hat{\varphi} \in \operatorname{Aut}_{A}\left(\operatorname{Ext}_{R}^{p}(A, R)\right)$ is given by the multiplication by the inverse of the Berezinian of the automorphism $\operatorname{Ber}(\varphi)^{-1}$, 


$$
\begin{aligned}
\widehat{\varphi}: \operatorname{Ext}_{R}^{p}(A, R) & \longrightarrow \operatorname{Ext}_{R}^{p}(A, R) \\
\mathcal{D} & \longmapsto \operatorname{Ber}(\varphi)^{-1} \cdot \mathcal{D}
\end{aligned}
$$

Proof Let us fix the base $\left\{x_{1}, \ldots, x_{p} \mid \theta_{1}, \ldots, \theta_{q}\right\}$ for $V$. Then, $\varphi \in A u t_{A}(V)$ is represented by an invertible matrix $[M] \in G L(p \mid q, A)$

$$
[M(\varphi)]_{\alpha \beta}=\left(\begin{array}{c|c}
A & B \\
\hline C & D
\end{array}\right)=\left(\begin{array}{l|l}
a_{h i} & b_{h j} \\
\hline c_{k i} & d_{k j}
\end{array}\right)
$$

where $A \in G L\left(p, A_{0}\right), D \in G L\left(q, A_{0}\right)$ are even and $B \in \operatorname{Hom}\left(A^{p}, A^{q}\right), C \in \operatorname{Hom}\left(A^{q}, A^{p}\right)$ are odd submatrices, such that one has the following transformations

$$
\begin{aligned}
& x_{i}^{\prime}:=\varphi\left(x_{i}\right)=\sum_{h=1}^{p} x_{h} a_{h i}+\sum_{k=1}^{q} \theta_{k} c_{k i}, \\
& \theta_{j}^{\prime}:=\varphi\left(\theta_{j}\right)=\sum_{h=1}^{p} x_{h} b_{h j}+\sum_{k=1}^{q} \theta_{k} d_{k j} .
\end{aligned}
$$

We recall that if $\varphi_{i}$ for $i=1,2$ are automorphisms of $V$, we have a contravariant functorial construction (see the remarks around (4.8)), such that $\left(\varphi_{1} \circ \varphi_{2}\right)^{*}=\phi_{2}^{*} \circ \varphi_{1}^{*}: \mathcal{K}^{*} \rightarrow \mathcal{K}_{\text {. }}^{*}$, therefore the product of two matrices $M\left(\varphi_{1}\right) \cdot M\left(\varphi_{2}\right) \in G L(p \mid q, A)$ corresponds to the product of two elements $\hat{\varphi}_{M\left(\varphi_{2}\right)} \cdot \hat{\varphi}_{M\left(\varphi_{1}\right)}$ acting as automorphisms of $\operatorname{Ext}_{R}^{p}(A, R)=H^{p}\left(\mathcal{K}^{*}\right)$. We can thus use the decomposition

$$
\left(\begin{array}{c|c}
A & B \\
\hline C & D
\end{array}\right)=\left(\begin{array}{c|c}
1 & B D^{-1} \\
\hline 0 & 1
\end{array}\right)\left(\begin{array}{c|c|c}
A-B D^{-1} C & 0 \\
\hline 0 & D
\end{array}\right)\left(\begin{array}{c|c}
1 & 0 \\
\hline D^{-1} C & 1
\end{array}\right)
$$

to reduce ourselves to the following cases.

$$
\text { (1): } M=\left(\begin{array}{l|l}
A & 0 \\
\hline 0 & D
\end{array}\right), \quad \text { (2) }: M=\left(\begin{array}{l|l}
1 & 0 \\
\hline * & 1
\end{array}\right), \quad \text { (3) }: M=\left(\begin{array}{l|l}
1 & * \\
\hline 0 & 1
\end{array}\right) \text {. }
$$

Let us consider the class of the generator of the homology, we call it $\mathcal{D}=\theta_{1} \ldots \theta_{q} \otimes \partial_{\chi_{1}} \ldots \partial_{\chi_{p}} \in \operatorname{Ext}_{R}^{p}(A, R)$ as above, and let us examine its transformation under automorphisms of the form (1), (2), (3) separately.

(1) In this case, it is simply to see that $\mathcal{D}$ transforms as $\operatorname{det}(D) \cdot \operatorname{det}(A)^{-1}$, as one has factorization of the transformations of the $\theta$ 's - contributing with $\operatorname{det}(D)$ and of the $\partial_{\chi}$ 'scontributing with $\operatorname{det}(A)^{-1}$.

(2) In this case one can observe that a generic automorphism of this forms is a composition of elementary automorphisms of the forms

$$
\begin{array}{ll}
x_{i}^{\prime}=x_{i}+\alpha \theta_{k}, & \alpha \in A_{1} \\
x_{l}^{\prime}=x_{l}, & l \neq i,
\end{array}
$$




$$
\theta_{j}^{\prime}=\theta_{j}
$$

It follows that one finds the following transformations:

$$
\begin{gathered}
\partial_{x_{l}}=\partial_{x_{l}^{\prime}}, \quad \forall l \\
\partial_{\theta_{j}}=\partial_{\theta_{j}^{\prime}}, \quad j \neq k \\
\partial_{\theta_{k}}=\partial_{\theta_{k}^{\prime}}+\alpha \partial_{x_{i}^{\prime}} .
\end{gathered}
$$

Thus, recalling that $\partial_{\chi_{i}}=\partial_{\pi x_{i}}$ and that $\partial_{\ell_{j}}=\partial_{\pi \theta_{j}}$, these are rewritten as

$$
\begin{gathered}
\partial_{\chi_{l}}=\partial_{\chi_{l}^{\prime}}, \quad \forall l \\
\partial_{\ell_{j}}=\partial_{\ell_{j}^{\prime}}, \quad j \neq k \\
\partial_{\ell_{k}}=\partial_{\ell_{k}^{\prime}}+\alpha \partial_{\chi_{i}^{\prime}} .
\end{gathered}
$$

In particular, one sees that $\mathcal{D}$ is invariant under these transformation.

(3) This is similar to the previous case. Indeed it can be observed again that a generic automorphism of this form is a composition of elementary automorphisms of the forms

$$
\begin{array}{ll}
x_{i}^{\prime}=x_{i} & \\
\theta_{j}^{\prime}=\theta_{j}+\beta x_{k}, \quad & \beta \in A_{1}, \\
\theta_{m}^{\prime}=\theta_{m}, & m \neq j .
\end{array}
$$

Thus, similarly, one finds that

$$
\begin{gathered}
\partial_{\chi_{k}}=\beta \partial_{\ell_{j}^{\prime}}+\partial_{\chi_{k}^{\prime}}, \\
\partial_{\chi_{i}}=\partial_{\chi_{i}^{\prime}}, \quad i \neq k \\
\partial_{\ell_{j}}=\partial_{\ell_{j}^{\prime}} \quad \forall j .
\end{gathered}
$$

It follows that the transformations reads

$$
\begin{aligned}
\theta_{1} \ldots \theta_{q} \otimes \partial_{\chi_{1}} \ldots \partial_{\chi_{p}}= & \theta_{1}^{\prime} \ldots \theta_{q}^{\prime} \otimes \partial_{\chi_{1}^{\prime}} \ldots \partial_{\chi_{p}^{\prime}}+ \\
& +(-1)^{j+1} \beta x_{k} \theta_{1} \ldots \hat{\theta}_{j} \ldots \theta_{q} \otimes \partial_{\chi_{1}^{\prime}} \ldots \partial_{\chi_{p}^{\prime}}+ \\
& +(-1)^{k+1} \theta_{1}^{\prime} \ldots \theta_{q}^{\prime} \otimes \partial_{\ell_{j}} \partial_{\chi_{1}} \ldots \hat{\partial}_{\chi_{k}} \ldots \partial_{\chi_{p}}
\end{aligned}
$$

where the element marked with a hat is missing. Noticing that the second and the third elements are of lower degree either in the $\theta$ 's or in the $\partial_{\chi}$ 's with respect to the generator 
$\mathcal{D} \in \operatorname{Ext}_{R}^{p}(A, R)$, hence they do not contribute to the transformation of the homology class, which is again invariant.

Combining the above cases, it follows that one has that $\hat{\varphi}_{M(\varphi)}=\operatorname{det}(D) \operatorname{det}\left(A-B D^{-1} C\right)^{-1}$, which is nothing but $\operatorname{Ber}(M(\varphi))^{-1}$ as claimed.

The previous theorem shows that an automorphism $\varphi_{V} \in A u t_{A}(V)$ induces an automorphism $\hat{\varphi} \in A u t_{A}\left(\operatorname{Ext}_{R}^{p}(A, R)\right)$ that is given by the multiplication of the inverse of the Berezinian of the trasformation,

$$
\begin{aligned}
& \widehat{\varphi}_{V}: \operatorname{Ext}_{S y m \bullet V}^{p}(A, S y m \bullet V) \longrightarrow \operatorname{Ext}_{S y m \bullet V}^{p}(A, S y m \bullet V) \\
& \mathcal{D} \longmapsto \operatorname{Ber}\left(\varphi_{V}\right)^{-1} \cdot \mathcal{D} \text {. }
\end{aligned}
$$

Clearly, if one considers instead of $V$ its $d u a l V^{*}=\operatorname{Hom}_{A}(V, A)$ and the related homology of the dual of the super Koszul complex one finds

$$
\begin{gathered}
\widehat{\varphi}_{V^{*}}: \operatorname{Ext}_{S y m^{\bullet} V^{*}}^{p}\left(A, \operatorname{Sym}_{\bullet} V^{*}\right) \longrightarrow \operatorname{Ext}_{S y m^{\bullet} V^{*}}^{p}\left(A, S y m^{\bullet} V^{*}\right) \\
\mathcal{D}^{*} \longmapsto \operatorname{Ber}\left(\varphi_{V}\right) \cdot \mathcal{D}^{*} .
\end{gathered}
$$

This remark naturally leads to the following definition.

Definition 5.2 (Berezinian of a Free $A$-Module) Let $V$ be a free $A$-supermodule of rank $p \mid q$ for $A$ any superalgebra. Then we call the Berezinian of $V$ the free $A$-supermodule of rank $\delta_{0,(p+q) \bmod 2} \mid \delta_{1,(p+q) \bmod 2}$ given by

$$
\operatorname{Ber}(V):=\operatorname{Ext}_{R}^{p}(A, R) \cong \Pi^{p+q} A,
$$

where $R:=S y m_{A}^{*} V^{*}$ and $A=R / I_{\max }$ for $I_{\max }:=\bigoplus_{k \geq 0} S y m^{k} V^{*}$.

Then, by the previous remark, an automorphism $\varphi: V \rightarrow V$ induces an automorphism $\operatorname{Ber}(\varphi): \operatorname{Ber}(V) \rightarrow \operatorname{Ber}(V)$ which is given by the multiplication by $\operatorname{Ber}(\phi)$ and such that $\operatorname{Ber}\left(\varphi_{1} \circ \varphi_{2}\right)=\operatorname{Ber}\left(\varphi_{2}\right) \operatorname{Ber}\left(\varphi_{1}\right)$.

As a conclusive remark, let us stress that this construction "parallels" in superalgebra the ordinary construction of the determinant or canonical module of a free module $V$ via homology of its related Koszul complex. In this sense, it should be clear the deep meaning behind the "slogan" that the Berezinian replaces the determinant when passing from a commutative setting to a supercommutative setting: notice further that the provided construction of the Berezinian module via super Koszul complex reduces to the construction of the ordinary determinant module via Koszul complex if $V$ purely even, i.e. it is of rank $p \mid 0$.

Furthermore, notice that the construction is readily generalizable from algebra to geometry, just by substituting the Ext-module with the $\mathcal{E} x t$-sheaf. Indeed, over a supermanifold $\mathcal{M}:=\left(|\mathcal{M}|, \mathcal{O}_{\mathcal{M}}\right)($ see $[1,5,8])$ the structure sheaf $\mathcal{O}_{\mathcal{M}}$ plays the role of $A$, while the free supermodule $V$ becomes a locally-free sheaf $\mathcal{E}$ of rank $p \mid q$ over $\mathcal{M}$ (see [3]). In particular, the choice of $\mathcal{E}:=\Omega_{\mathcal{M}}^{1}$, the cotangent sheaf of $\mathcal{M}$, leads to what is usually called the Berezinian sheaf of $\mathcal{M}$, i.e. $\operatorname{Ber}(\mathcal{M}):=\mathcal{E x t}_{\operatorname{Sym}^{*}\left(\Omega_{\mathcal{M}}^{1}\right)^{*}}\left(\mathcal{O}_{\mathcal{M}}, \operatorname{Sym} m^{\bullet}\left(\Omega_{\mathcal{M}}^{1}\right)^{*}\right)$, locally generated by 


$$
\mathcal{D}=d x_{1} \ldots d x_{p} \otimes \partial_{\theta_{1}} \ldots \partial_{\theta_{q}} \in \operatorname{Ber}(\mathcal{M}):=\mathcal{E x t}_{\operatorname{Sym}^{*}\left(\Omega_{\mathcal{M}}^{1}\right)^{*}}^{p}\left(\mathcal{O}_{\mathcal{M}}, \operatorname{Sym}\left(\Omega_{\mathcal{M}}^{1}\right)^{*}\right)
$$

if $\left\{x_{1}, \ldots, x_{p} \mid \theta_{1}, \ldots, \theta_{q}\right\}$ are local coordinates for $\mathcal{M}$.

The notion of Berezinian sheaf of a supermanifold is well-established in the literature. In this regards, a nice intrinsic construction of the Berezinian of a smooth supermanifold of dimension $p \mid q$ has been given by Hernández Ruipérez and Muñoz Masque in [11], as a certain quotient of the (locally free) sheaf of degree $q$ differential operators taking values in (compactly supported) $p$-forms. In particular, in section 3 of [11], the authors discuss the equivalence of their result with the (categorial) construction given by Penkov in [10] in the context of $\mathcal{D}_{\mathcal{M}}$-modules theory on supermanifolds. An explicit realization of Penkov's construction has been recently given by these authors in [2] (see Section 3, Theorem 3.6), where the Berezinian sheaf, together with all the other sheaves of integral forms, emerges as the homology of a complex of sheaves of modules over a non-commutative sheaf. In particular, the (dual of the) Koszul complex, as discussed in this note, is a quotient of the construction in [2], thus making contact between the realization given by Hernández Ruipérez and Muñoz Masque in [11] and that in this paper, via Penkov's construction.

It is worth stressing, though, that it is not obvious how to provide a direct connection between the present construction of the Berezinian sheaf via Koszul complex-which holds true in the smooth category but also in the holomorphic and algebraic categoryand that given in [11], which actually requires working in the smooth category only, since compactly supported functions play a crucial role in the construction. More precisely, with reference to [11], it is not possible to trace back in the above Koszul complex construction a submodule playing the role of $\mathscr{K}$ in the quotient sheaf discussed by Hernández Ruipérez and Muñoz Masque. Nonetheless, the construction in [11] is relatively easier compared to that given in this note and it has the perk of making explicit the relation with Berezin integral over smooth supermanifolds (see Theorem 2.3 in [11]).

Funding Open Access funding enabled and organized by Projekt DEAL.

Open Access This article is licensed under a Creative Commons Attribution 4.0 International License, which permits use, sharing, adaptation, distribution and reproduction in any medium or format, as long as you give appropriate credit to the original author(s) and the source, provide a link to the Creative Commons licence, and indicate if changes were made. The images or other third party material in this article are included in the article's Creative Commons licence, unless indicated otherwise in a credit line to the material. If material is not included in the article's Creative Commons licence and your intended use is not permitted by statutory regulation or exceeds the permitted use, you will need to obtain permission directly from the copyright holder. To view a copy of this licence, visit http://creativecommons.org/licenses/by/4.0/.

\section{References}

1. Bartocci, C., Bruzzo, U., Hernández-Ruipérez, D.: The geometry of supermanifolds. Springer (1991)

2. Cacciatori, S.L., Noja, S., Re, R.: The unifying double complex on supermanifolds. arXiv:2004.10906

3. Carmeli, C., Fioresi, R., Varadarajan, V.S.: Super bundles. Universe 4 (2018)

4. Catenacci, R., Cremonini, C., Grassi, P.A., Noja, S.: On forms, cohomology, and BV Laplacians in odd symplectic geometry. Lett. Math. Phys. 111, 44 (2021)

5. Carmeli, C., Caston, L., Fioresi, R.: Mathematical foundations of supersymmetry. EMS (2001)

6. Deligne, P. et al.: Quantum field theory and strings: a course for mathematicians. Vol 1, AMS (1999)

7. Eisenbud, D.: Commutative algerbra - with a view toward Algebraic Geometry. Springer, New York (1995)

8. Manin, Yu.I.: Gauge Fields and Complex Geometry. Springer, New York (1988) 
9. Ogievetskii, O.V., Penkov, I.B.: Serre duality for projective supermanifolds. Funct. Anal. its Appl. 18, 68-70 (1984)

10. Penkov, I.B.: $\mathscr{D}$-Modules on Supermanifolds. Invent. Math. 71, 501-512 (1983)

11. Hernández Ruipérez, D., Muñoz Masque, J.: Construction Intrinsique du faisceau de Berezin d'une varietè graduèe. C. R. Acad. Sc. Paris 301, 915-918 (1985)

Publisher's Note Springer Nature remains neutral with regard to jurisdictional claims in published maps and institutional affiliations. 